\title{
Evaluación y predicción del work engagement en voluntarios: el papel del sentido de la coherencia y la reevaluación cognitiva
}

\author{
Eva Garrosa $^{1 *}$, Luis M. Blanco-Donoso ${ }^{1}$, Bernardo Moreno-Jiménez ${ }^{1}$, \\ Ana González ${ }^{2}$, Mónica Fraca ${ }^{3}$ y María J. Meniz ${ }^{3}$ \\ ${ }^{1}$ Facultad de Psicologia. Dpto. Psicología Biológica y de la Salud. Universidad Autónoma de Madrid. \\ ${ }^{2}$ Dpto. Psicooncología. Asociación Española Contra el Cáncer \\ ${ }^{3}$ Dpto. Voluntariado. Asociación Española Contra el Cáncer
}

\begin{abstract}
Resumen: Una perspectiva salutogénica se está imponiendo en el ámbito de las organizaciones. Frente al estudio del desgaste profesional o burnout, y en cierta medida, gracias a él, ha surgido el concepto de vinculación al trabajo o engagement. Este trabajo plantea conocer el nivel de engagement de los voluntarios de una asociación que atiende a personas con cáncer y a sus familiares, y el rol que juegan la Reevaluación Cognitiva y el Sentido de Coherencia en su explicación. También se analiza la manera en la que estos factores cognitivos y emocionales se relacionan a la hora de explicar la vinculación dentro de la organización. La muestra está compuesta por 136 voluntarios. Se llevaron a cabo análisis descriptivos, de correlación, de regresión jerárquica y análisis de efectos indirectos a través de la técnica de remuestreo o bootstrapping. Los resultados muestran que la reevaluación cognitiva tiene un efecto directo en la varianza del engagement, así como el sentido de coherencia, que a su vez ejercería un papel mediador entre ambas variables. Se discuten las implicaciones de los resultados para promover la vinculación al trabajo de los voluntarios.

Palabras clave: Bootstrapping; efecto indirecto; engagement; reevaluación
\end{abstract} cognitiva; sentido de la coherencia; vinculación con el trabajo; voluntarios.
Title: Assesment and prediction of work engagement in a health volunteers sample: the role of Sense of Coherence and Cognitive Reappraisal. Abstract: A salutogenic perspective within organizations is becoming more important. Faced with the study of burnout, and to some extent, thanks to its, has emerged the concept work engagement. This study aims to determine the level of engagement of volunteers from an association that serves people with cancer and their families, and show some factors that could explain it, in particular, cognitive reappraisal and sense of coherence. We also want to analyze the way in which cognitive and emotional factors related to explain this relationship within the organization. The sample consists of 136 volunteers. Descriptive analysis, correlation analysis, regression analysis and analysis of indirect effects were conducted using the statistical procedure bootstrapping. The results show that cognitive reappraisal has a direct effect on the variance of engagement. The sense of coherence, besides having a direct effect, have a mediating role between the two variables. We discuss the implications of the results to promote the work engagement of volunteers.

Key words: Bootstrapping; cognitive reappraisal; engagement; indirect effect; sense of coherence; volunteers.

\section{Introducción}

En los últimos veinticinco años se ha investigado y desarrollado ampliamente el estudio del estrés laboral y sus consecuencias sobre las organizaciones y sobre los individuos que las componen. Bajo ésta perspectiva, apareció y se potenció la línea de investigación sobre el burnout o desgaste profesional (Maslach y Jackson, 1986). Han sido numerosas las investigaciones, comunicaciones y trabajos presentados en congresos dirigidos a estudiar éste problema (Gálvez, Moreno-Jiménez y Mingote 2009).

Éste fenómeno ha sido identificado en muchos profesionales de la salud (Felton, 1998), incluida el área de la medicina oncológica, donde se ha encontrado una alta prevalencia (Camps et al., 2009; Whippen y Canellos, 1991). La costosa tarea de trabajar con pacientes enfermos y sus familiares y trabajar con asuntos emocionalmente complicados, hacen que los retos que asumen diariamente estos profesionales se conviertan en factores de riesgo que pueden predisponer a la aparición del desgaste profesional (Trufelli et al., 2008; Moriana, 2006).

Los voluntarios que trabajan en el área de la salud también están expuestos a estas demandas emocionales (Freudenberger, 1974). Sin embargo, el gran interés que los inves-

\footnotetext{
* Dirección para correspondencia [Correspondence address]: Eva Garrosa Hernández. Facultad de Psicologia. Dpto. Psicología Biológica y de la Salud. Universidad Autónoma de Madrid. Ciudad Universitaria de Cantoblanco. C/ Iván Pavlov, 6. 28049, Madrid (España). Email: eva.garrosa@uam.es
}

tigadores han mostrado por este colectivo ha sido menor en comparación con el colectivo profesional (Chacón, Vecina, Barrón y de Paúl, 1999; Lewig, Xanthopoulou, Bakker, Dollard y Metzer, 2007). Estudios previos en nuestro país con voluntarios pertenecientes al área de la salud han mostrado como la incidencia de éste síndrome es infrecuente en ellos (Chacón y Vecina, 2000; Fernández, Yélamos y Olivares, 2004; Montesinos, 2006). En general, los voluntarios se encuentran satisfechos con su actividad como voluntario (Montesinos, Martínez y Fernández, 2008), especialmente aquellos que desarrollan su actividad dentro del área socioasistencial (Dávila de León y Díaz Morales, 2005).

Frente al estudio del burnout, y en cierta medida, gracias a éste, desde la perspectiva de la psicología positiva surgió el concepto de work engagement, o, vinculación laboral (Maslach y Leiter, 1997; Schaufeli, Salanova, González - Roma y Bakker, 2002). Schaufeli et al. (2002, p.74) definen y conceptualizan el engagement como "un estado de la mente positivo relacionado con el trabajo que se caracteriza por el vigor, la dedicación y la absorción”. Estas tres dimensiones componen la estructura de dicho constructo. El vigor es conceptualizado como altos niveles de energía y fuerza mental mientras se trabaja, con la voluntad de invertir esfuerzo en lo que se está haciendo y persistir ante las dificultades. La dedicación se refiere a estar fuertemente involucrado en la actividad que uno realiza, experimentando un sentimiento de significado, entusiasmo, inspiración, orgullo y reto. Y por último, la $a b-$ sorción, se caracteriza por estar plenamente concentrado y dejándose llevar por la tarea, percibiendo que el tiempo transcurre rápidamente (Schaufeli et al., 2002). Bakker y Derks 
(2010) representan la conceptualización del burnout y del engagement en un modelo circumplejo de emociones (Russell y Carroll, 1999), donde la vinculación con el trabajo esta profundamente relacionada con emociones como el entusiasmo, la felicidad, la satisfacción y la energía. Shirom (2011), en alusión al vigor, hace una revisión de cómo frecuentemente en la literatura se ha entendido a éste como un estado afectivo positivo relacionado con el trabajo, más allá que una mera emoción positiva específica.

El engagement se ha relacionado con diversas consecuencias organizacionales, entre ellas, la aparición de conductas fuera del rol establecido (Halbesleben y Wheeler, 2008), la intención de abandonar la organización, el compromiso organizacional (Vecina, Chacón, Sueiro y Barrón, 2012; Schaufeli y Bakker, 2004), con la calidad del servicio reportado por los clientes (Salanova, Agut y Pieró, 2005) y con la presencia de emociones positivas (Salanova, Llorens y Schaufeli, 2011).

En los últimos años, el engagement está siendo un foco considerable de investigación en el ámbito profesional (Schaufeli et al., 2002), aunque hasta donde nosotros conocemos son escasos los estudios realizados dentro de un contexto de voluntariado de la salud (Vecina et al., 2012). Dentro de este contexto se han estudiado otros conceptos como el compromiso organizacional, la permanencia en la organización, y la satisfacción con la actividad como voluntario (Chacón, Vecina y Dávila, 2007; Vecina, Chacón y Sueiro, 2009). Vecina y cols. (2012) han evaluado recientemente la estructura interna del concepto work engagement en una muestra de voluntarios provenientes del área social y medioambiental. Estos autores, además de confirmar la estructura de tres factores del constructo, muestran como los voluntarios presentan un nivel alto de engagement, especialmente aquellos más veteranos. Fourie, Rothmann y van de Vijver (2008) también encontraron resultados similares.

El estudio del engagement es consecuencia de un creciente interés en psicología de la salud organizacional por los aspectos positivos que favorecen la promoción y el mantenimiento de la salud en los miembros de las organizaciones (Bakker y Schaufeli, 2008; Bakker, Rodriguez-Muñoz y Derks, 2012; Nelson y Simmons, 2003; Turner, Barling y Zachartos, 2002). Su estudio aporta una nueva comprensión acerca de los procesos positivos que emergen en las personas y grupos que las componen (Bakker y Derks, 2010). Durante mucho tiempo el foco de la psicología se ha centrado en los aspectos negativos de las personas (Seligman y Csikszentmihalyi, 2000) y ésta visión ha influenciado en la dinámica de las organizaciones. Desde éste punto de partida, la psicología positiva y la teoría organizacional confluyen en un nuevo acercamiento denominado Psicología de la Salud Ocupacional Positiva (POHP), centrada en el estudio de las condiciones psicológicas positivas y fortalezas humanas que están relacionadas con el bienestar de los miembros de una organización (Schaufeli, 2004).

El voluntariado es una pieza clave dentro de nuestra sociedad y constituye un elemento fundamental para la consecución de los objetivos de muchas organizaciones. En estos momentos, las organizaciones cuentan con un serio problema de permanencia de sus voluntarios, y es importante conocer qué elementos hacen que su vinculación con la organización aumente (Lewig et al., 2007), viéndose en investigaciones previas la importancia que juegan en este sentido la satisfacción con la actividad, las variables personales y la presencia de emociones positivas (González, Leiva, Sepúlveda y Vega, 2004; Vecina et al., 2012; Vecina y Chacón, 2005).

Los voluntarios que trabajan en el campo de la salud se encuentran con que tienen que manejar altas demandas emocionales en su día a día (Chacón y Vecina, 2000; Freudenberger, 1974). Para ello es necesario contar con recursos que permitan hacer frente a estas demandas para no sólo no desgastarse, sino también tener experiencias de enriquecimiento y bienestar (Lewig et al., 2007).

Antonovsky (1979) plantea un modelo salutogénico (Figura 1) centrado en aquellos aspectos de las personas que favorecen la salud y el bienestar, es decir, centrado en los factores protectores y de desarrollo personal. Acorde con esta visión, la naturaleza humana está caracterizada por la omnipresencia de estresores y lo que pretende determinar a través de este modelo es cómo las personas se mantienen en el polo positivo del bienestar (Antonovsky, 1979). En esta línea, los Recursos Generales de Resistencia al Estrés (GRRs) son un elemento fundamental dentro de este modelo. Se refieren a las características de una persona, un grupo, o una situación que facilitan el hacer frente a los estresores y demandas inherentes a la vida. Estos crean experiencias que se caracterizan por la coherencia, la participación y el equilibrio.

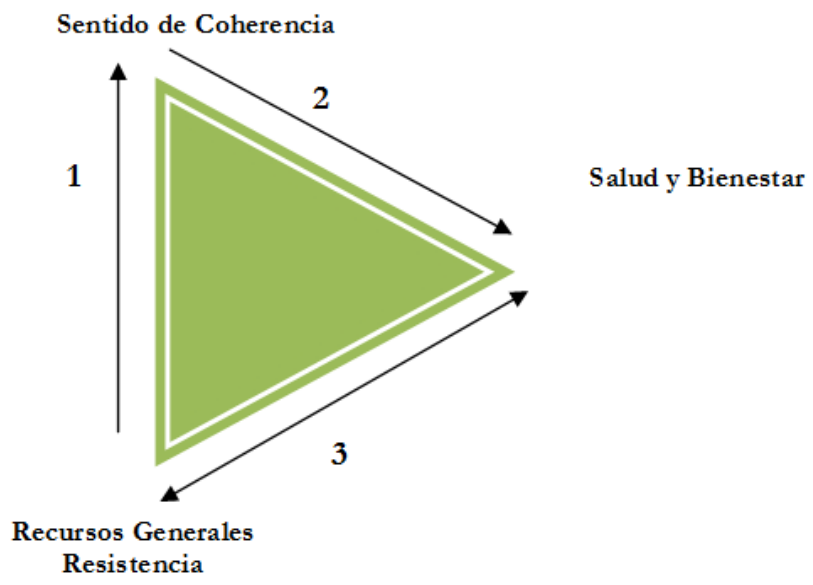

Figura 1. Modelo Salutogénico de Antonovsky (1987)

Ejemplos de estos recursos son las variables de personalidad, los estilos de afrontamiento, el apoyo y posición social, el dinero, y la autoestima, entre otros (Antonovsky, 1979). Estos recursos ayudan a las personas a construir experiencias de vida coherentes (Antonovsky, 1987; Marsh, Clinkinbird, Thomas y Evans, 2007; Wiesmann y Hanninch, 2010). Así, los recursos de resistencia favorecen la creación de un sentido de coherencia, que es lo que verdaderamente explicaría la salud y el bienestar de las personas. Un adecuado manejo de las altas demandas emocionales por parte de los voluntarios 
podría constituir un recurso de resistencia que pueda favorecer experiencias coherentes de vida, que deriven al mismo tiempo en un estado de engagement en su actividad como voluntario (Figura 2).

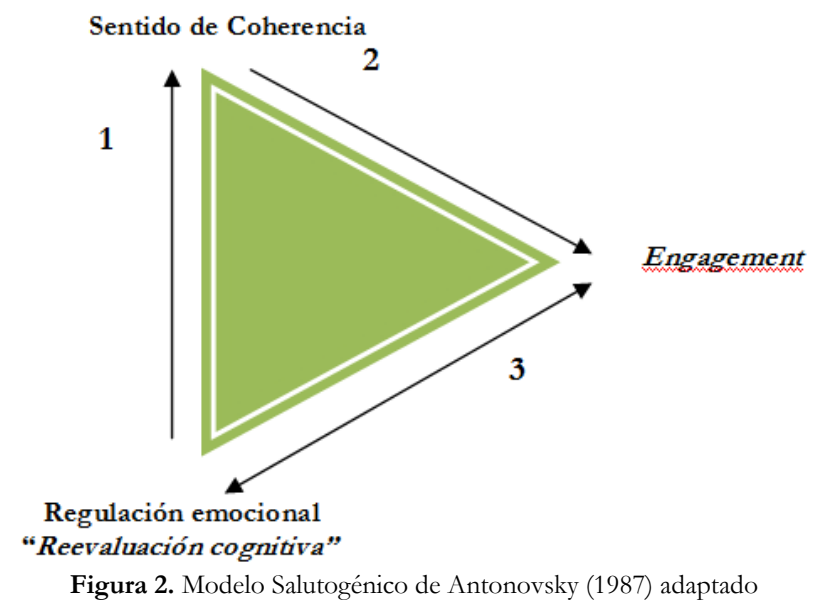

El Sentido de Coherencia (SOC) hace referencia a "la percepción global de sentimientos de dominio y confianza en que los estímulos derivados del ambiente externo e interno en el curso de la vida son estructurados, predecibles y explicables, de que los recursos personales que uno posee son adecuados para enfrentarnos a las demandas presentadas por estos estímulos, y que esas demandas constituyen un reto digno de invertir tiempo, energía y compromiso en ello" (Antonovsky, 1987, p.19). Este constructo está formado por tres dimensiones denominadas comprensibilidad, manejabilidad y significatividad. La comprensibilidad es considerada como el componente cognitivo del constructo. Los individuos con una elevada comprensibilidad perciben los estímulos a los que se enfrentan dotándolos de un marco cognitivo y considerando la información como ordenada, consistente, estructurada y clara en vez de caótica, desordenada, azarosa, accidental o impredecible. La manejabilidad es entendida como el componente instrumental del constructo, y hace referencia al grado en que los individuos entienden que los recursos adecuados para hacer frente a las demandas del medio se encuentran a su disposición. Por último, la significatividad es considerada la dimensión emocional del constructo, e implica una visión comprometida de ciertas áreas de la vida para las cuales merece la pena emplear tiempo y esfuerzo (Antonovsky, 1987). Las personas con un fuerte SOC muestran más bienestar (Ericksson y Lindström, 2007), y mayor afecto positivo (Strumpfer, Gouws y Viviers, 1998).

El SOC es una variable que cobra una especial importancia en el contexto en el que se ha desarrollado nuestra investigación y con las restantes variables estudiadas, demostrando jugar un papel central en la predicción de la vinculación con el trabajo (Bezuidenhou y Cilliers, 2010; Fourie et al., 2008; May, Gilson y Harter, 2004). Parece que las personas con una elevada significatividad en sus vidas tienden con mayor probabilidad a definir los estresores como retos a los que dar la bienvenida e invertir energía en ellos (Feldt, 1997). Schaufeli y Bakker (2001) señalan que el engagement, específicamente el vigor, se caracteriza por cierta resiliencia y voluntad de invertir energía y esfuerzo en el trabajo que uno desempeña. Sin embargo, cuando uno carece de esa significatividad, corre el riesgo de desgastarse profesionalmente (Pines, 1993). El SOC es una variable proveedora de significado a lo que uno hace, y podría contribuir a la aparición y fortalecimiento del engagement de los voluntarios (Fourie et al., 2008; Strümpfer, 2003). De la misma manera podría generar un marco cognitivo del lugar de trabajo que permita concebir éste como claro, estructurado y predecible, a la vez que manejable y con acceso y control a todos los recursos disponibles para llevar a cabo la actividad del voluntario.

En la literatura científica, podemos encontrar diversos recursos que han sido vinculados con la formación de un elevado SOC (Antonovsky, 1979; Cohen, 1997; Johnson, 2004; Read, Aunola, Feldt, Leinonen y Ruoppila, 2005; Volanen, Lahelma, Silventoinen y Suominen, 2004; Wiesmann y Hanninch, 2010). Pallant y Lae (2002) encontraron que la autoestima, el optimismo y el locus de control construían y daban forma al SOC. Las estrategias para regular adecuadamente las emociones pueden ser también un recurso crucial para que los voluntarios se sientan bien y más comprometidos con su actividad. Al mismo tiempo, regular adecuadamente las emocionales, dándoles diferentes sentidos y significados puede crear experiencias de coherencia y significado (Park y Folkman, 1997) que hagan más fácil desarrollar su actividad, y que les hagan sentir más enérgicos, dedicados y absortos en sus tareas. De esta manera podríamos estar hablando de que la regulación emocional podría tener un efecto directo e indirecto sobre el engagement de los voluntarios.

Gross (1998) propone un modelo de regulación emocional que considera ciertas estrategias de regulación como más propensas a producir salud y bienestar. Este autor propone diversas estrategias, entre las que se encuentran la reevaluación y la supresión emocional. La reevaluación cognitiva es la modificación del significado y la interpretación que se da a un evento o situación. Se trata de "una estrategia de bajo costo que se implementa en el inicio del proceso de la generación de las emociones, antes de que surjan, modificando así su impacto sobre el individuo" (Gross, 1998, p. 275). Esta estrategia está asociada con muchos indicadores positivos (Gross y John, 2003; Nezlek y Kuppens, 2008), incluyendo el rendimiento en la tarea en diferentes contextos (Raftery y Bizer, 2009; Wallace, Edwards, Shull y Finch, 2009), y considerándose como una de las estrategias de regulación emocional más eficaces en la reducción de las emociones negativas (John y Gross, 2004; Nezlek y Kuppens, 2008).

Gross se refiere a los "reevaluadores" o "supresores" (Gross, Richards y John, 2006) para referirse a aquellas personas que tienden a utilizar una estrategia determinada en sus vidas, suponiendo cierta estabilidad en su uso, pero al mismo tiempo considerando que estas estrategias pueden ser 
aprendidas y utilizadas por cualquier persona en algún momento. También se producen cambios en su uso a lo largo de la vida, al igual que en el SOC (Antonovsky, 1987). Por tanto, la reevaluación cognitiva dentro del modelo de Gross (1998), abarcaría mucho más que un estilo de afrontamiento situacional (Lazarus y Folkman, 1984) y podría ser considerada un recurso de resistencia generalizada que contribuiría a crear un sentido de coherencia en las personas. Park y Folkman (1997) consideran que la revaluación tiene un papel fundamental en la creación de congruencia entre el significado de las demandas y nuestro sentido de vida global.

El modelo salutogénico de Antonovsky (1979) podría dar respuesta al hecho de que haya voluntarios dentro de las organizaciones de la salud que a pesar de las elevadas demandas emocionales de su trabajo, en lugar de desgastarse, se encuentren muy vinculados con su actividad gracias a sus variables personales, manteniendo niveles altos de energía, dedicación y absorción. Dentro del ámbito laboral, el modelo de demandas y recursos (Demerouti, Bakker, Nachreiner y Schaufeli, 2001) da cuenta de los distintos procesos que pueden acontecer en los individuos de una organización en la predicción del burnout y el engagement. A partir de la distinta combinación entre las demandas laborales y los recursos de distinta naturaleza, incluidas las variables personales, se desarrollan los dos procesos opuestos (Lewig et al., 2007; Xanthopoulou, Bakker, Demerouti y Schaufeli, 2007).

Las variables de personalidad ejercen un rol importante a la hora de generar la posibilidad de aparición del burnout frente al engagement y viceversa (Garrosa, Moreno-Jiménez, Rodriguez-Carvajal, y Rodriguez-Muñoz, 2011). Han sido estudiadas variables clásicas como el neuroticismo y la extraversión en este sentido (Bakker, Van der Zee, Lewig y Dollard, 2006; Langelaan, Bakker, Van Doornen y Schaufeli, 2006), y también, desde una perspectiva salutogénica, variables como la Resiliencia, Personalidad Resistente y SOC. También se ha podido observar que las emociones positivas y negativas, influyen sobre el desgaste, la vinculación y la conducta de ayuda de los voluntarios (Isen y Levin, 1972; Vecina y Chacón, 2005). Todas estas variables podrían actuar como potenciales predictores del bienestar dentro de las organizaciones, a la vez que moderar las relaciones existentes entre las variables organizacionales y sus consecuencias (Bezuidenhou y Cilliers, 2010; Fourie et al., 2008; Garrosa, Moreno-Jiménez, Liang y González, 2008; Ladstätter, Garrosa, Badea y Moreno, 2010; Naudé y Rothmann, 2006; Nelson y Simmons, 2003; Strümpfer, 2003; Strümpfer y De Bruin, 2009; Van der Colff y Rotmann, 2009).

Tomando como referencia el Modelo Salutogénico de Antonovsky (1979), se pretende estudiar la contribución de los aspectos cognitivo-emocionales en la predicción del engagement en voluntarios. Ante las demandas de la organización y de la tarea, las variables personales podrían predecir que un voluntario se vincule con su actividad, se sienta lleno de energía, dedicado y absorto en ella, frente a desgastarse. Con esta investigación se pretende estudiar el papel que desempeñaría la estrategia de reevaluación cognitiva (Gross,
1998) y el SOC (Antonovsky, 1987) en la predicción del engagement. De la misma manera, trata de comprobar si la relación existente entre reevaluación y engagement se produce en parte por el SOC como se muestra en la Figura 2, de tal manera que las dos primeras variables se relacionarían a través de esta tercera. Desde el modelo de Antonovsky supondría considerar la reevaluación cognitiva (Gross, 1998) como un recurso generalizado de resistencia que da forma al SOC, lo que realmente explicaría el engagement. Cuando una persona tiende a utilizar la reevaluación como modo habitual de regular sus emociones, es posible que construya una percepción más comprensible de la realidad que tiene presente, que perciba más accesibles los recursos de su organización y que le dote de un significado que haga que merezca la pena dedicar tiempo y energía a las actividades que le rodean, lo que generará realmente el estado de engagement en su actividad como voluntario (Britt, Adler y Bartone, 2001).

\section{Método}

\section{Participantes}

En este estudio participaron un total de 150 voluntarios (115 mujeres, 35 hombres) con edades comprendidas entre los 18 años y los 89 años $(M=52.36$ y $D T=17.08)$ que colaboraban voluntariamente con la Asociación Española Contra el Cáncer (aecc) en tareas asistenciales y de cuidado con una antigüedad comprendida desde los 3 meses a los 20 años $(M=5.01$ y $D T=4.90)$ y procedentes de 9 instituciones $\mathrm{y}$ centros sanitarios distintos (Hospital Infanta Sofía, Hospital de Henares, Hospital Clínico, Hospital Ramón y Cajal, Hospital 12 de Octubre, Hospital Gregorio Marañón, Hospital Puerta de Hierro, Hospital de Alcorcón, y Sede provincial de Madrid de la aecc).

\section{Variables e Instrumentos de medida}

Se utilizó la escala UWES (Utrecht Work Engagement Scale) en su versión de 15 items que mide work engagement o vinculación con el trabajo (Schaufeli et al., 2002). Este cuestionario mide las tres dimensiones del constructo, que son, vigor (p.e: "en mi trabajo me siento lleno de energía"), dedicación (p.e: "mi trabajo me inspira”) y, absorción (p.e: "cuando estoy trabajando olvido todo lo que pasa alrededor de mí"). Cada ítem se responde en una escala del 0 "nunca" al 6 "siempre". El cuestionario ha sido adaptado y validado a la población española, mostrando unas adecuadas propiedades psicométricas (Salanova, Schaufeli, Llorens, Peiró y Grau, 2001).

Las Escalas de Afectividad (PANAS, de las siglas de Positive and Negative Affect Schedule) ha sido desarrollada por Watson, Clark y Tellegen (1988) como un instrumento de medida de dos dimensiones del afecto: afecto positivo y afecto negativo entendiendo como afecto positivo (AP) la dimensión de emocionalidad positiva, energía y afiliación, y al afecto negativo (AN) como un factor que expresa la sen- 
sibilidad temperamental a estímulos negativos. Los reactivos consisten en palabras que describen diferentes sentimientos y emociones que la persona ha experimentado en la última semana y en general o regularmente, y a las que la persona califica, de acuerdo al grado en que las experimenta, en una escala de cinco puntos en donde 1 significa "muy poco o nada", y 5 "extremadamente". En nuestro estudio únicamente hemos tomado la medida general de la afectividad positiva como variable control, dado el carácter positivo de las variables utilizadas y según recomiendan algunos autores (Mojza, Sonnentag y Bornemann, 2011). Esta versión de PANAS ha demostrado validez y fiabilidad que apoyan su uso en población española (Robles y Páez, 2003).

El Cuestionario de Regulación Emocional (ERQ, de las siglas Emotion Regulation Questionnaire), es un instrumento que evalúa dos dimensiones de la regulación emocional: reevaluación y supresión (Gross y John, 2003); entendiendo como reevaluación la reinterpretación de un potencial estímulo emocional en un estímulo no emocional, y la supresión como la inhibición de la conducta expresiva durante la activación emocional, en términos generales a la hora de abordar nuestras emociones (Gross, 1998). De acuerdo a los objetivos de nuestra investigación, se seleccionó únicamente la escala de reevaluación. Los ítems que conforman la escala son 6 e indican el proceso emocional implicado, por ejemplo "Controlo mis emociones cambiando la manera de pensar sobre la situación en la que me encuentro", en una escala donde 1 significa "totalmente en desacuerdo" y el 7 "totalmente de acuerdo". Se utilizó la versión adaptada y validada al castellano por Rodriguez-Carvajal, Moreno-Jiménez y Garrosa (2006) que muestra tener unas propiedades psicométricas adecuadas en la población española.

El Cuestionario de Orientación a la Vida (OLQ, de las siglas Orientation to Life Questionnaire), es un instrumento que mide el SOC (Antonovsky, 1987). Evalúa las tres dimensiones que conforman este constructo, siendo éstas comprensibilidad, manejabilidad y significatividad. Está formado por 29 items, en los que los participantes puntúan en una escala de 7 puntos tipo Likert. Ejemplos de éstos ítems son: “ En la pasada década, mi vida ha estado...1= llena de cambios sin saber lo que ocurría , o $7=$ completamente clara y coherente" (comprensibilidad); "Cuando realiza algo que le hace sentirse bien, es cierto que... 1= algo ocurrirá para estropear este sentimiento, o, 7= me seguiré sintiendo bien" (manejabilidad); "anticipa que su vida personal en el futuro estará...1=totalmente vacía de objetivos, o , 7= llena de objetivos y significado"(significatividad). El cuestionario ha sido adaptado y validado para la población española mostrando unas adecuadas propiedades psicométricas (MorenoJiménez, Alonso y Álvarez, 1997).

Finalmente se recogieron datos sociodemográficos como el sexo, la edad y la antigüedad de los voluntarios dentro de la organización. Estas variables se utilizaron como variables control en el análisis de regresión debido a la gran variabilidad de la muestra y por su relación con las variables dependientes en estudios previos (Vecina et al., 2012).

\section{Procedimiento}

Fueron convocados los voluntarios de la Asociación a través de los coordinadores de cada programa y centro de voluntariado para cumplimentar los cuestionarios en una única jornada que duró toda una mañana. Previamente fueron informados de los objetivos de la investigación y del papel que desempeñarían en ella. Con ello dieron su consentimiento a través de un documento firmado. La totalidad de los instrumentos fueron rellenados por los participantes, que iban acompañados de una hoja que recogía datos sociodemográficos como la edad, sexo, y los años de antigüedad en la asociación. Una vez lo completaron se les informó de una posterior reunión informativa con los primeros resultados del estudio. Posteriormente se excluyeron de éste 14 cuestionarios que no estaban correctamente completados, para conformar la base de datos con la que finalmente se trabajaría y se llevarían a cabo los correspondientes análisis de datos.

\section{Análisis de datos}

La muestra con la que se trabajó finalmente estuvo compuesta por 136 participantes. Se llevó a cabo un análisis descriptivo de las variables obteniendo las medias y desviaciones típicas de todas ellas. Para ver la relación entre las variables se realizó un análisis de correlaciones Pearson. Posteriormente se llevó a cabo un análisis de regresión jerárquica con el objetivo de estudiar la contribución a la varianza explicada de las variables predictoras (reevaluación, comprensibilidad, manejabilidad y significatividad) sobre las variables criterio (vigor, dedicación y absorción), controlando las variables sociodemográficas y el afecto positivo. Esta última variable se utilizó para minimizar los efectos de la multicolinealidad dado el carácter positivo asociado con el engagement (Podsakoff, MacKenzie, Lee, y Podsakoff, 2003). Finalmente, para comprobar si existían efectos indirectos de las dimensiones del SOC que resultaron predictivas en el análisis de regresión, sobre la relación existente entre reevaluación y las tres dimensiones de engagement, se aplicó la técnica de remuestreo o bootstrapping.

Ésta técnica se recomienda para estudiar los efectos indirectos de terceras variables en casos dónde éstas no se ajustan a la distribución normal o se desconoce y cuándo se trabaja con muestras relativamente pequeñas, superando de este modo las limitaciones del procedimiento clásico (Ato y Vallejo, 2011; Baron y Kenny, 1986; Preacher y Hayes, 2004). Se ha utilizado en diversas investigaciones en psicología con este fin (Estévez y Calvete, 2009; Gaudiano, Herbert y Hayes, 2010). En esta técnica, los datos muestrales son tratados como si constituyesen los datos de toda la población, es decir se utilizan como el universo del que se extraerán muestras con reemplazamiento. En este estudio se ha utilizado un bootstrap o muestra reemplazada de $N=$ 1000. Para cada remuestreo se calculará el valor del estima- 
dor bootstrap que se utilizará para estimar la variabilidad muestral, así como el cálculo de intervalos de confianza y la realización de contrastes de hipótesis. Bajo este procedimiento, se hablaría de que existen efectos indirectos si una vez calculado el intervalo de confianza el valor 0 se encuentra fuera de este intervalo (Preacher y Hayes, 2004).

\section{Resultados}

En la Tabla 1 se reflejan las medias, desviaciones típicas y alfa de cronbach de todas las variables estudiadas. Todas las escalas, excepto manejabilidad, mostraron unos índices alfa adecuados $(\alpha>.70)$ (Nunnally, 1978). Como puede observarse, las medias de las tres dimensiones del engagement tienden a situarse en valores próximos, siendo la más elevada la correspondiente a la dimensión dedicación.

En la tabla 1 también se muestran las correlaciones bivariadas entre las variables escogidas en nuestro estudio. La edad correlaciona negativamente con la dimensión manejabilidad $(r=-.29, p<.01)$ y con la dimensión significatividad $(r=$ $.25, p<.01)$. La antigüedad no ha mostrado tener ninguna relación significativa con el resto de variables. El afecto positi- vo se relaciona de manera significativa con las tres dimensiones de engagement, exactamente con vigor $(r=.46, p<.01)$, dedicación $(r=.40, p<.01)$ y con absorción $(r=.44$, $p<.01)$. Las tres dimensiones del SOC correlacionan de manera significativa y en sentido positivo con las tres dimensiones del engagement, de tal manera que comprensibilidad se relaciona con $v i$ gor $(r=.26, p<.01)$, con dedicación $(r=.31, p<.01)$ y con $a b$ sorción $(r=.20, p<.05)$; manejabilidad y vigor $(r=.37, p<.01)$, dedicación $(r=.40, p<.01)$ y con absorción $(r=.32, p<.01) ; \mathrm{y}$ por último significatividad con vigor $(r=.39, p<.01)$, dedicación $(r=.47, p<.01)$ y con absorción $(r=.37, p<.01)$. La relación entre la estrategia de regulación reevaluación cognitiva y las dimensiones del SOC es positiva, pero únicamente significativa en el caso de significatividad ( $r=.18, p<.05$ ). Sin embargo, sí que correlaciona positiva y de manera estadísticamente significativa con vigor $(r=.28, p<.01)$, con dedicación $(r=.32$, $p<.01)$ y con absorción $(r=.31, p<.01)$.

Por último, considerando las tres dimensiones del constructo de engagement, todas ellas correlacionan de manera positiva y significativamente entre ellas.

Tabla 1. Medias, desviaciones típicas, correlaciones y alpha de Cronbach.

\begin{tabular}{|c|c|c|c|c|c|c|c|c|c|c|c|c|}
\hline & $M$ & $D T$ & 1 & 2 & 3 & 4 & 5 & 6 & 7 & 8 & 9 & 10 \\
\hline 1. Edad & & 52.3 & $17.0-$ & & & & & & & & & \\
\hline 2. Antigüedad & & 5.01 & 4.90 .42 & - & & & & & & & & \\
\hline 3. Afecto positivo & & 3.60 & $.64-.03$ & -.14 & $(.80)$ & & & & & & & \\
\hline 4. Reevaluación Cognitiva & & 4.74 & 1.28 .04 & .06 & .10 & $(.83)$ & & & & & & \\
\hline 5. Comprensibilidad & & 4.39 & 1.14-.05 & .07 & .12 & .13 & $(.76)$ & & & & & \\
\hline 6. Manejabilidad & & 4.88 & $0.71-.29^{* *}$ & -.02 & .13 & .16 & $.57^{* *}$ & $(.59)$ & & & & \\
\hline 7.Significatividad & & 5.32 & $1.22-.25^{* *}$ & -.01 & .12 & $.18^{*}$ & $.72^{* *}$ & $.70^{* *}$ & $(.84)$ & & & \\
\hline 8. Vigor & & 4.53 & .91 .00 & -.04 & $.46^{* *}$ & $.28^{* *}$ & $.26^{* *}$ & $.37^{* *}$ & $.39^{* *}$ & $(.83)$ & & \\
\hline 9.Dedicación & & 4.65 & $1.11-.14$ & -.05 & $.40^{* *}$ & $.32^{* *}$ & $.31^{* *}$ & $.40^{* *}$ & $.47^{* *}$ & $.70^{* *}$ & $(.83)$ & \\
\hline 10. Absorción & & 4.45 & $.99-.07$ & -.00 & $.44^{* *}$ & $.31^{* *}$ & $.20^{*}$ & $.32^{* *}$ & $.37^{* *}$ & $.81^{* *}$ & $.83^{* *}$ & $(.81)$ \\
\hline
\end{tabular}

$*$ Correlación significativa $p<.05$ (2-tailed). $* *$ Correlación significativa $p<.01$ (2-tailed).

Notas: alpha Cronbach entre paréntesis en la diagonal.

Para estudiar los efectos principales de las variables reevaluación cognitiva y las tres dimensiones del SOC sobre las tres dimensiones de engagement, se llevaron a cabo tres análisis de regresión jerárquica por pasos para cada variable resultado. En todos los análisis se introdujeron variables sociodemográficas como el género, la edad, la antigüedad como voluntario y el afecto positivo como variables control. En el segundo paso se introdujo la variable reevaluación cognitiva. Por último, en el tercer paso, se introdujeron las tres dimensiones de SOC (comprensibilidad, manejabilidad y significatividad). Se comprobó que los valores FIV y los índices de tolerancia se encontraran dentro del rango recomendado para descartar la colinealidad de las variables predictoras, siendo el valor FIV menor de $10 \mathrm{y}$ el índice de tolerancia mayor a .10.

Como se aprecia en la Tabla 2, las variables control edad y el afecto positivo explican el $22.3 \%$ de la varianza total de vigor. $[F(4)=8.169, p<.001]$. La estrategia de reevaluación añade un $4.9 \%$ de varianza explicada $[F(1)=7.544, p<.01]$, y las dimensiones del SOC un $13.5 \%[F(3)=8.312, p$
$<.001]$, específicamente manejabilidad $(\beta=.247, p<.05)$ y significatividad ( $\beta=.314, p<.05$ ). Comprensibilidad no mostró efecto significativo. Al introducir la estrategia reevaluación cognitiva en el modelo 2 resultó ser significativa $\beta=.223$, $p$ $<.01)$, pero al aparecer en la tabla únicamente los coeficientes del modelo de regresión final, cuando se introdujeron las variables del SOC, su significación desapareció $(\beta=.147, p$ $>$.05).

Respecto a la variable dedicación, la variable afecto positivo vuelve a ser predictiva $(\beta=.281, p<.001)$, explicando un $16.8 \%$ de su varianza explicada $[F(4)=5.638, p<.001]$. La inclusión de la reevaluación cognitiva resultó ser significativa $[F(1)=12.347, p<.001]$ y añadió un $8.3 \%$ de varianza adicional, mostrando también su importancia a la hora de predecir dedicación $(\beta=.217, p<.01)$. Y por último, únicamente la variable significatividad fue predictiva de las tres dimensiones del SOC $(\beta=.394, p<.01)$, añadiendo un $15.1 \%$ de varianza al modelo e indicando que su inclusión en éste fue también significativa $[F(3)=9.093, p<.001]$. 
Por otra parte, el afecto positivo como control y tal como se suponía vuelve a ser una variable predictiva para el caso de absorción $(\beta=.387, p<.001)$. Su inclusión en el modelo resultó significativa $[F(4)=7.745, p<.001]$, aportando un $21.4 \%$ de varianza a éste. La reevaluación cognitiva también resultó ser predictiva $(\beta=.212, p<.01)$, aportando un $7.4 \%$ de varianza adicional, y mostrándose significativa su inclusión en el modelo $[F(1)=11.693, p<.001]$. Finalmente comprensibilidad y significatividad añadieron un $10.5 \%$ de varianza $[F(3)=6.359, p<.001]$, resultando significativas en la predicción de la variable $(\beta=.26, p<.05),(\beta=.350, p$ $<.01)$.

Tabla 2. Análisis de regresión jerárquica ${ }^{a}$

\begin{tabular}{|c|c|c|c|c|c|c|}
\hline \multirow[b]{2}{*}{ Variables } & \multicolumn{2}{|c|}{ Vigor } & \multicolumn{2}{|c|}{ Dedicación } & \multicolumn{2}{|c|}{ Absorción } \\
\hline & $\Delta R^{2}$ & $\beta$ & $\Delta R^{2}$ & $\beta$ & $\Delta R^{2}$ & $\beta$ \\
\hline Paso 1 & $.223^{* * *}$ & & $.168^{* * *}$ & & $.214^{* * *}$ & \\
\hline $\begin{array}{l}\text { Género } \\
\text { Edad } \\
\text { Antigüedad } \\
\text { Afecto positivo }\end{array}$ & & $\begin{array}{l}-.077 \\
.193^{*} \\
-.040 \\
.383^{* * *}\end{array}$ & & $\begin{array}{l}-.044 \\
-.006 \\
.009 \\
.281^{\text {**** }}\end{array}$ & & $\begin{array}{l}-.048 \\
.053 \\
.065 \\
.387^{\text {**** }}\end{array}$ \\
\hline Paso 2 & $.049^{* *}$ & & $.083^{* * *}$ & & $.074^{* * *}$ & \\
\hline $\begin{array}{l}\text { Reevaluación } \\
\text { Cognitiva }\end{array}$ & & .147 & & $.217^{* *}$ & & $.212^{* *}$ \\
\hline Paso 3 & $.135^{* * *}$ & & $.151^{* * *}$ & & $.105^{* * *}$ & \\
\hline $\begin{array}{l}\text { Comprensibilidad } \\
\text { Manejabilidad } \\
\text { Significatividad }\end{array}$ & & $\begin{array}{l}.184 \\
.247^{*} \\
.314^{*}\end{array}$ & & $\begin{array}{l}.138 \\
.154 \\
.394^{* *}\end{array}$ & & $\begin{array}{l}.260^{*} \\
.189 \\
.350^{* *}\end{array}$ \\
\hline $\mathrm{R}^{2}$ & .406 & & .402 & & .393 & \\
\hline $\mathrm{R}^{2}$ corregido & .363 & & .358 & & .349 & \\
\hline
\end{tabular}

${ }^{a}$ Coeficientes de regresión estandarizados del modelo final ${ }^{*} p<.05 .{ }^{* *} p<.01 .{ }^{* * *} p<.001$

Se llevó a cabo un estudio de efectos indirectos para comprobar si las dimensiones del SOC que habían resultado ser significativas en el análisis de regresión mediaban la relación existente entre reevaluación cognitiva y engagement. Para ello se trabajó con un macro de sintaxis en SPSS provisto por Preacher y Hayes (2004). Los resultados de los análisis bajo este procedimiento se interpretan bajo intervalos de confianza y se muestran en la tabla 3 .

Como puede observarse en el caso de la reevaluación cognitiva y vigor, los resultados de la prueba bootstrapping muestran que el 0 se encuentra fuera del intervalo de confianza de $95 \%$ para las variables de manejabilidad y significatividad, indicando un posible efecto indirecto significativo $(p<.05)$. No se testó la variable comprensibilidad por no haber resultado predictiva en el análisis de regresión.

Cuando tratamos la relación entre reevaluación cognitiva y dedicación teniendo en cuenta la dimensión significatividad, encontramos que los valores del intervalo de confianza dejan fuera el valor 0 , indicando de nuevo un posible efecto indirecto significativo $(p<.05)$. Las otras dos dimensiones del
SOC no se incluyeron por no haber resultado significativas en el análisis de regresión.

Tabla 3. Intervalos de confianza para los efectos indirectos de las dimensiones del SOC en el vigor, la dedicación y absorción, empleando el procedimiento de bootstrapping.

\begin{tabular}{|c|c|c|c|c|}
\hline \multirow{2}{*}{ Vigor } & \multirow[t]{2}{*}{$\begin{array}{c}\text { Efectos } \\
\text { indirectos }\end{array}$} & \multirow[t]{2}{*}{$\begin{array}{c}\text { Punto } \\
\text { estimado (ET) }\end{array}$} & \multicolumn{2}{|c|}{$\begin{array}{l}95 \% C I \\
\text { Inferior } \\
\text { Superior }\end{array}$} \\
\hline & & & & \\
\hline & Total & $.055(.031)$ & .002 & .132 \\
\hline Manejabilidad & & $.031(.020)$ & .003 & .103 \\
\hline Significatividad & & $.041(.031)$ & .001 & .136 \\
\hline \multicolumn{5}{|l|}{ Dedicación } \\
\hline \multirow[t]{2}{*}{ Significatividad } & Total & $.068(.040)$ & .006 & .174 \\
\hline & & $.061(-.041)$ & .006 & .187 \\
\hline \multicolumn{5}{|l|}{ Absorción } \\
\hline Comprensibilidad & Total & $.026(.026)$ & -.106 & .008 \\
\hline Significatividad & & $.049(.035)$ & .004 & .158 \\
\hline
\end{tabular}

\section{Nota: 1000 muestras bootstrap}

De manera similar nos encontramos con que significatividad vuelve a presentar un efecto indirecto significativo $(p$ $<.05$ ) en la relación existente entre reevaluación cognitiva y absorción, pues se observa que el 0 se encuentra fuera del intervalo de confianza de $95 \%$. Un efecto indirecto que sin embargo no mostró la variable comprensibilidad, al situarse el 0 dentro del intervalo de confianza $(p>.05)$.

\section{Discusión}

En este trabajo se ha analizado el nivel de engagement que presentan los voluntarios pertenecientes a una asociación dedicada a la asistencia de pacientes oncológicos. También se ha evaluado el poder predictivo de dos variables empíricamente asociadas con el bienestar, reevaluación cognitiva y $S O C$, en relación con las tres dimensiones que conforman el constructo de engagement. Y por último, se comprobó si la reevaluación cognitiva se relaciona con el engagement a través del efecto indirecto del SOC. El estudio, por tanto, supone una aproximación innovadora a los recursos generales de resistencia que desde el modelo de Antonovsky (1979) pueden explicar el engagement de los voluntarios en oncología, específicamente el efecto indirecto del SOC en la relación positiva que se establece entre la reevaluación cognitiva y el engagement, aspectos que aportan una nueva línea de trabajo centrada en la profundización de los mecanismos intervinientes en el desarrollo del engagement en los voluntarios. A través de los procesos de regulación y el SOC, se contribuye en la operativización del desarrollo de programas de intervención centrados en los mecanismos que de manera directa e indirecta puedan tener su efecto en aspectos más profundos y estables de la personalidad que se relacionan con el bienestar.

En relación con el primer objetivo, los voluntarios presentan un nivel moderado en vigor (3.21-4.80) y dedicación (3.01- 4.90), y alto en absorción (4.41-5.35), si son comparados con una muestra normativa de trabajadores españoles (Salanova y Schaufeli, 2009). Los voluntarios de este estudio 
presentan moderados niveles de energía y fuerza mental mientras desarrollan su tarea, están involucrados en la actividad que realizan y frecuentemente se encuentran concentrados y dejándose llevar por ésta, percibiendo que el tiempo transcurre rápidamente. Estos resultados se suman a la evidencia aportada por otros estudios que informan de niveles moderadamente altos de engagement en voluntarios (Fourie et al., 2008; Vecina et al., 2012), lo que podría ser relevante si tenemos en cuenta que este estado de vinculación se ha asociado con la presencia de emociones positivas (Salanova et al., 2011), con la calidad del servicio informado por los usuarios (Salanova et al., 2005), y con el compromiso organizacional (Vecina et al., 2012; Schaufeli et al., 2004).

En relación con nuestro segundo objetivo, significatividad mostró ser la variable más predictiva del vigor, la dedicación y la absorción de los voluntarios. Estos resultados van en la línea de lo encontrado por otros autores (Bezuidenhout et al., 2010; Strümpfer,2002), mostrando como esta variable juega un rol central en la predicción de la vinculación con el trabajo. Aquellos voluntarios que tienden a ver los cambios que se producen en su vida como dignos de invertir esfuerzo y energía, probablemente afrontarán con mayor vigor sus actividades dentro de la organización. Como ya apuntaban Schaufeli y Bakker (2001), el vigor se caracteriza por cierta resiliencia y voluntad de invertir energía y esfuerzo en la actividad que uno desempeña. Además, si un voluntario le proporciona significado a su vida, probablemente influya también en cómo se involucre en aquello que elige hacer, mostrándose con mayor dedicación y absorción en ello. El SOC no deja de ser una variable proveedora de significado que podría influir en la aparición y mantenimiento del engagement de los voluntarios (Stümpfer, 2002). Manejabilidad mostró ser sólo predictiva en el caso del vigor, y no para las otras dos. Percibir su actividad como un conjunto de tareas y experiencias que son tolerables, que pueden afrontar con los recursos personales que uno tiene o con el acceso legítimo a los recursos de los otros, puede contribuir a que los voluntarios se sientan con mayor energía y fuerza mental, y en definitiva con mayor vigor. No obstante, estos resultados deben ser tratados con cautela, pues la escala que se utilizó para medir esta dimensión mostró un alfa de cronbach bajo ( $a$ $<.70$ ). Según Bezuidenhout y cols., (2010), el componente de manejabilidad establece un balance equilibrado de carga, entre nuestros recursos y las demandas, y ello puede contribuir a no sentirnos agotados, sino por el contrario, con energía. Quizá por ello que no haya mostrado un efecto significativo en las demás dimensiones del engagement, donde quizá influyen junto con los aspectos emocionales otros aspectos más cognitivos. Este es el caso de la comprensibilidad, la última dimensión en analizar, que mostró ser únicamente predictiva a la hora de sentirse absorto en la tarea. Parece que para que un individuo llegue a manifestar absorción en ésta, además de que la persona disfrute con ella y la provea de significado, es importante que la conciba clara y predecible. Por el contrario, percibir que la mayoría de estímulos son poco definidos y sin sentido, puede llevar al voluntario a que no se sien- ta plenamente concentrado, y mucho menos a que perciba que el tiempo transcurre rápidamente. Esta variable, al tener una naturaleza más cognitiva que emocional, puede no tener un impacto tan significativo sobre las dimensiones de vigor y dedicación, quizá de naturaleza más emocional y que implican aspectos más motivacionales dentro del individuo (Bezuidenhout et al., 2010; Strümpfer, 2002).

En cuanto a la variable reevaluación cognitiva, mostró ser significativa en la explicación de la dedicación y de la absorción. En el caso del vigor, si bien es cierto que su inclusión en el modelo resultó significativa, lo dejó de ser cuando se incluyó en éste las tres dimensiones del SOC. Manejar adecuadamente nuestras emociones se ha relacionado con el bienestar (Karademas, Tsalikou y Tallarou, 2011) y con el rendimiento en diferentes contextos (Raftery et al., 2009; Wallace et al., 2009). Específicamente reevaluar cognitivamente nuestras emociones ayuda al mantenimiento de un mayor balance de emociones positivas (Augustine y Hemenover, 2009). Reinterpretar una situación de manera que cambie su impacto emocional hace que disminuya la intensidad de las emociones negativas, y ello puede aumentar el nivel de engagement. Por el contrario, el no reevaluar las emociones puede dar lugar a que perdure durante más tiempo la emoción negativa y sus consecuencias, teniéndose el individuo que esforzar después para reducir esa emoción (Gross, 1998), e impidiendo que se involucre en su verdadera actividad, que esté entusiasmado e inspirado (dedicación) y que se centre y deje llevar por ella (absorción). El bajo poder predictivo de la reevaluación frente a otras variables como significatividad podría indicar el mayor peso de los aspectos emocionales frente a los cognitivos en la vinculación de los voluntarios con su trabajo.

En todos los análisis realizados, se ha observado el efecto significativo del afecto positivo y las dimensiones de engagement, lo que demuestra la necesidad de su utilización como variable control para disminuir los efectos de la multicolinealidad producida por la estrecha asociación entre el engagement y las emociones positivas (Bakker, y Derks, 2010; Schaufeli et al., 2001). Igualmente, también pone de manifiesto que las emociones son un elemento importante a considerar en la relación que se establece entre todas estas variables (Salanova et al., 2011; Shirom, 2011). El papel de las emociones positivas en los voluntarios ha sido relacionado con su intención de permanencia dentro de la organización (Vecina y Chacón, 2005).

En relación al tercer objetivo, resulta relevante para conocer su efecto como recursos de resistencia al estrés en el modelo de Antonovsky (1979) y profundizar en los mecanismos intervinientes en la relación con el engagement. Desde esta perspectiva este estudio trata de analizar si los voluntarios que reevalúan sus emociones y cambian cognitivamente la situación que las genera para sentirse mejor, se sienten con mayor vigor, dedicación y absorción en parte gracias a que la reinterpretación cognitiva contribuya a proporcionar un marco coherente de la situación a la persona, dotándola de claridad (comprensibilidad); a una percepción de acceso a los 
recursos para afrontar las demandas (manejabilidad); y un sentido y significado a lo que uno hace, digno de invertir energía y esfuerzo en ello (significatividad). Los resultados de los análisis muestran cómo la variable significatividad media la relación entre reevaluación y las tres dimensiones de engagement; igualmente manejabilidad lo hizo también en el caso del vigor. Estos datos van en la línea de lo encontrado por Britt y cols. (2001), que demuestran como la evaluación cognitiva positiva de una situación puede generar significatividad, claridad y manejabilidad y en consecuencia un mejor ajuste con la eficacia de resultados. Una vez más, la variable significatividad muestra tener un rol central en la predicción del engagement.

En conclusión, los voluntarios mostraron un nivel moderado y alto en las distintas dimensiones de engagement. Este nivel se explica en su mayor parte por la dimensión significatividad del voluntario, influyendo también, aunque con menor fuerza y no en todas las dimensiones, el manejo emocional de las situaciones a través de la reevaluación cognitiva. Esta reevaluación contribuye al mismo tiempo a generar significados que darían lugar a una mayor vinculación. La percepción en el voluntario de que las actividades a las que dedica tiempo son dignas de invertir energía y esfuerzo explica en buena medida su vinculación con ellas (Schaufeli y Bakker, 2001), incluso más que la manera de cómo las maneja emocionalmente. La decisión de ser voluntario y estar vinculado con su actividad en el tiempo con energía, dedicación y absorción es una decisión personal y motivacional profundamente conectada con el significado que los voluntarios dan a su vida y a las actividades que emprenden, y por eso quizá su destacado valor predictivo frente a otras variables, como se ha señalado anteriormente (Bezuidenhout et al., 2010; Strümpfer, 2002). El SOC tiene una conceptualización donde la persona trata de encontrar sentido a sus acciones y creemos que éste es un factor clave que explica la realización del voluntariado, su carácter social y comunitario.

Este estudio cuenta con algunas limitaciones, por ejemplo el número de participantes que constituye la muestra, la obtención de datos mediante autoinforme y la imposibilidad de establecer relaciones causa-efecto. Sería conveniente en futuras investigaciones ampliar el número de participantes en el estudio, para ello, sería importante insistir en la relevancia y el reconocimiento de la función de los voluntarios en la sociedad, así como, de la importancia de este tipo de

\section{Referencias}

Antonovsky, A. (1979). Health, stress and coping. San Francisco: Jossey-Bass.

Antonovsky, A. (1987). Unravelling the mystery of health: How people manage to stay well. San Francisco, USA: Jossey-Bass Publishers.

Ato, M., y Vallejo, G. (2011). Los efectos de terceras variables en la investigación psicológica. Anales de Psicología, 27(2), 550-561.

Augustine, A. A., y Hemenover, S. H. (2009). On the relative effectiveness of affect regulation strategies: A meta-analysis. Cognition and Emotion, 23(6), 1181-1220.

Bakker, A. B., y Derks, D. (2010). Positive occupational health psychology. En S. Leka y Houdmont J. (Eds.), Occupational Health Psychology (pp. 194224). Malden, MA: Blackwell Publishing. estudios para mejorar el bienestar de los voluntarios, e igualmente, sería necesario incluir a voluntarios procedentes de otras asociaciones dentro del ámbito de la salud para ampliar el estudio. También sería interesante realizar un estudio longitudinal e incluir otras variables resultado como la permanencia en la organización para poder establecer relaciones causales y los factores antecedentes del proceso de engagement. No obstante, este estudio tiene como novedad el evaluar el nivel de engagement en una muestra de voluntariado de la salud y estudiar qué factores de la persona son importantes en su predicción, así como su interacción. Además, desde este punto de vista, son pocos los estudios que han relacionado las tres dimensiones del constructo SOC y sus efectos directos e indirectos con engagement, dimensión por dimensión, en este tipo de colectivo, así como estudiar el papel de la estrategia cognitiva de reevaluación. Desde esta perspectiva el estudio pretende un acercamiento a los recursos generales de resistencia que mejora el bienestar en los voluntarios.

Las implicaciones prácticas de este estudio nos dirigen a la conveniencia de desarrollar fortalezas personales y de regulación en los voluntarios para no sólo prevenir consecuencias negativas dentro de la organización, sino además promover estados de vigor, dedicación y absorción en sus miembros. También puede contribuir a una mejor selección de los voluntarios que van a prestar atención a personas que se encuentran en una situación complicada. Además, los programas de voluntariado deben promover actividades que otorguen significado a las tareas que llevan a cabo los voluntarios, promover la percepción de recursos disponibles dentro de la organización en caso de necesitarlos y un marco cognitivo de la actividad que realizan y de sus limitaciones. Planes de formación específica dentro de esta área, así como grupos de apoyo al voluntariado dirigidos por supervisores profesionales serían importantes de cara a conseguir estos objetivos.

Los resultados de esta investigación contribuirán a aumentar el cuerpo de conocimiento existente en psicología de la salud ocupacional positiva en dirección al paradigma salutogénico.

Agradecimientos.- A los trabajadores y voluntarios de la Asociación Española Contra el Cáncer.

Bakker, A. B., Rodriguez-Muñoz, A., y Derks, D. (2012). La emergencia de la psicología de la salud ocupacional positiva. Psicothema, 24(1), 66-72.

Bakker, A. B., y Schaufeli, W. B. (2008). Positive organizational behavior: Engaged employees in flourishing organizations. Journal of Organizational Behavior, 29(2), 147-154.

Bakker, A. B., Van der Zee, K. I., Lewig, K. A., y Dollard, M. F. (2006). The relationship between the Big Five personality factors and burnout: A study among volunteer counselors. Journal of Social Psychology, 146(1), 3150 .

Baron R. M., y Kenny, D. A. (1986). The moderator-mediator variable distinction in social psychological research: Conceptual, strategic, and sta- 
tistical considerations. Journal of Personality and Social Psychology, 51(6), 1173-1182.

Bezuidenhout, A., y Cilliers, F. V. N. (2010). Burnout, work engagement and sense of coherence in female academics in higher-education institutions in South Africa. South African Journal of Industrial Psychology, 36(1), 11-22.

Britt, T. W., Adler, A. B., y Bartone, P. T. (2001). Deriving benefits from stressful events: The role of engagement in meaningful work and hardiness. Journal of Occupational Health Psychology, 6(1), 53-63.

Camps, C., Escobar, Y., Esteban, E., Almenárez, J. A., Moreno-Jiménez, B., Gálvez Herrer, M., Arranz, P., y Sánchez, P.T. (2009). Professional burnout among Spanish medical oncologists. Clinical and Translational Oncology, 11(2), 86-90.

Chacon, F., y Vecina, M. L. (2000). Motivation and burnout in volunteerism. Psychology in Spain, 4(1), 75-81.

Chacón, F., Vecina, M. L., Barrón, A., y de Pául, M. P. (1999). Burnout en voluntarios que trabajan con pacientes de SIDA o cáncer. Clínica y Salud, 10(2), 137-150.

Chacón, F., Vecina, M. L., y Dávila, M. C. (2007). The three-stage model of volunteers' duration of service. Social Behavior and Personality: an international journal, 35(5), 627-642.

Cohen, O. (1997). On the origins of a sense of coherence: Sociodemographic characteristics, or narcissism as a personality trait? Social Behavior and Personality, 25(1), 49-58.

Dávila, M. C. y Díaz, J. F. (2005). Voluntariado y Satisfacción vital. Revista de Intervención Psicosocial, 14 (1), 81-94.

Demerouti, E., Bakker, A. B., Nachreiner, F., y Schaufeli, W. B. (2001). The job demands resources model of burnout. Journal of Applied Psychology, $86(3), 499-512$.

Eriksson, M., y Lindström, B. (2007). Antonovsky's Sense of Coherence Scale and its relation with quality of life: A systematic review. Journal of Epidemiology and Community Health, 61(11), 938-944.

Estévez, A. y Calvete, E. (2009). Mediación a través de pensamientos automáticos de la relación entre esquemas y síntomas de depresión. Anales de Psicología, 2(1), 27-35.

Feldt, T. (1997). The role of sense of coherence in wellbeing at work: Analysis of main and moderator effects. Work \& Stress, 11(2), 134-147.

Felton, J. S. (1998). Burnout as a clinical entity its importance in health care workers. Occupational Medicine, 48(4), 237-250.

Fernández, B., Yélamos, C., y Olivares, M. E. (2004). Efectos derivados de la acción voluntaria: inventario de consecuencias de la acción voluntaria (ICAV). Psicooncología, 1(1), 113-126.

Fourie, L., Rothmann, S. y van de Vijver, F. J. R. (2008), A model of work wellness for non-professional counsellors in South Africa. Stress and Health, 24(1), 35-47.

Freudenberger, H. J. (1974). Staff burn-out. Journal of Social Issues, 30, 159165.

Gálvez Herrer, M., Moreno-Jiménez, B., y Mingote, J. C. (2009). El desgaste profesional médico. Revisión y guia de buenas prácticas. El vuelo de Ícaro. Madrid: Díaz de Santos.

Garrosa, E., Moreno-Jiménez, B., Liang, Y., y González, J. L. (2008). The relationship between socio-demographic variables, job stressors, burnout, hardly personality in nurses: a exploratory study. International Journal of Nursing Studies, 45 (3), 418-427.

Garrosa, E., Moreno-Jiménez, B., Rodríguez-Muñoz, A., y RodríguezCarvajal, R. (2011). Role stress and personal resources in nursing: A cross-sectional study of burnout and engagement. International Journal of Nursing Studies, 48(4), 479-489.

Gaudiano, B. A., Herbert, J. D., y Hayes, S. C. (2010). Is it the symptom or the relation to it? Investigating potential mediators of change in acceptance and commitment therapy for psychosis. Behavior Therapy, 41(4), 543-554.

González, L., Leiva Y., Sepúlveda, A., y Vega C. (2004). Factores psicológicos asociados a la permanencia y compromiso del voluntariado. Revista de Psicología, 13(2), 21-41.

Gross, J. J. (1998). The emerging field of emotion regulation: An integrative review. Review of General Psychology, 2(3), 271-299.

Gross, J. J., y John, O. P. (2003). Individual differences in two emotion regulation processes: Implications for affect, relationships, and wellbeing. Journal of Personality and Social Psychology, 85(2), 348-362.
Gross, J. J., Richards, J. M., y John, O. P. (2006). Emotion regulation in everyday life. En D.K. Snyder, J.A. Simpson, y J.N. Hughes (Eds.) Emotion regulation in families: Pathways to dysfunction and health, (pp. 13-35). Washington DC: American Psychological Association.

Halbesleben, J. R. B., y Wheeler, A. R. (2008). The relative roles of engagement and embeddedness in predicting job performance and intention to leave. Work \& Stress, 22(3), 242-256.

Isen, A. M. y Levin, P. A. (1972). Effect of feeling good an helping: Cookies and kindness. Journal of Personality and Social Psychology ,21(3),384-388.

John, O. P., y Gross, J. J. (2004). Healthy and unhealthy emotion regulation: Personality processes, individual differences, and life span development. Journal of Personality, 72(6),1301-1333.

Johnson, M. (2004). Approaching the salutogenesis of sense of coherence: The role of 'active' self-esteem and coping. British Journal of Health Psychology, 9(3), 419-432.

Karademas, E. C., Tsalikou, C., y Tallarou, M. (2011). The impact of emotion regulation and illness-focused coping strategies on the relation of illness-related negative emotions to subjective health. Journal of Health Psychology, 16(3), 510-519.

Ladstätter, F., Garrosa, E., Badea, C., y Moreno, B. (2010). Application of artificial neural networks to a study of nursing burnout. Ergonomics, 53(9), 1085-1096.

Langelaan, S., Bakker, A. B., Van Doornen, L. J. P., y Schaufeli, W. B. (2006). Burnout and work engagement: Do individual differences make a difference?. Personality and Individual Differences, 40(3), 521-532.

Lazarus, R. S., y Folkman, S. (1984). Stress, appraisal, and coping. New York: Springer.

Lewig, K. A., Xanthopoulou, D., Bakker, A. B., Dollard, M. F., y Metzer, J. C. (2007). Burnout and connectedness among Australian volunteers: A test of the job demands-resources model. Journal of Vocational Behavior, $71(3), 429-445$.

Marsh, S. C., Clinkinbird, S. S., Thomas, R. M., y Evans, W. P. (2007). Risk and protective factors predictive of sense of coherence during adolescence. Journal of Health Psychology, 12(2), 281-284.

Maslach, C., y Jackson, S. E. (1981). The measurement of experienced burnout. Journal Occupational behaviour, 2(2), 99-113.

Maslach, C., y Jackson, S. E. (1986). Maslach Burnout Inventory (2a .ed.). Palo alto, CA : Consulting Psychologists Press.

Maslach, C., y Leiter, M. P. (1997). The truth about burnout. San Francisco: Josey Bass Publishers.

May, D. R., Gilson, R. L., y Harter, L. M. (2004), The psychological conditions of meaningfulness, safety and availability and the engagement of the human spirit at work. Journal of Occupational and Organizational Psychology, 77(1), 11-37.

Mojza, E. J., Sonnentag, S. y Bornemann, C. (2011). Volunteer work as a valuable leisure-time activity: A day-level study on volunteer work, nonwork experiences, and well-being at work. Journal of Occupational and Organizational Psychology, 84(1), 123-152.

Montesinos F. (2006). Prevención del burnout en voluntariado de atención a pacientes de cáncer a través de ACT: un estudio exploratorio. VIII Congreso Internacional sobre el Estudio de la Conducta. Santiago de Compostela.

Montesinos, F., Martínez, A., y Fernández, B. (2008). Formación y Satisfacción del voluntariado de cuidados paliativos. Psicooncología, 5(2-3), 401408.

Moreno-Jiménez, B., Alonso, M., y Álvarez, E. (1997). Sentido de la coherencia, Personalidad resistente, autoestima y salud. Revista de psicología de la Salud, 9(2), 115-137.

Moriana, J. A. (2006). Burnout y cuidado de la salud en voluntarios. Revista electrónica de Intervención Psicosocial y Psicología Comunitaria, 1(2), 52-57.

Naudé, J.L.P., y Rothmann, S. (2006). Work-related well-being of emergency workers in Gauteng. South African Journal of Psychology, 36(1), 63-81.

Nelson, D. L., y Simmons, B. L. (2003). Health psychology and work stress: A more positive approach. En J. Campbell Quick, y L. E. Tetrick (Eds.), Handbook of Occupational Health Psychology, 1st ed, (pp. 97-119). Washington DC: American Psychological Association.

Nezlek, J. B., y Kuppens, P. (2008). Regulating Positive and Negative Emotions in Daily Life. Journal of Personality, 76(3), 561-580.

Nunnally, J. C. (1978). Psychometric Theory. Second Edition. New York: McGraw-Hill. 
Pallant, J. F., y Lae, L. (2002). Sense of coherence, well-being, coping and personality factors: Further evaluation of the Sense of Coherence Scale. Personal Individual Difference, 33(1), 39-48.

Park, C. L, y Folkman, S. (1997). Meaning in the context of stress and coping. Review of General Psychology, 1(2), 115-44.

Pines, A. M. (1993). Burnout. En L. Goldberger y S. Breznitz (Eds.). Handbook of stress. Theoretical and clinical aspects (2nd ed.) (pp. 386-402). New York: Free Press.

Podsakoff, P. M., MacKenzie, S. B., Lee, J., y Podsakoff, N. P. (2003). Common method biases in behavioral research: A critical review of the literature and recommended remedies. Journal of Applied Psychology, 88(5), 879-903.

Preacher, K. J., y Hayes, A. F. (2004). SPSS and SAS procedures for estimating indirect effects in simple mediation models. Behavior Research Methods, Instruments and Computers, 36(4), 717-731.

Raftery, J. N., y Bizer, G. Y. (2009). Negative feedback and performance: The moderating effect of emotion regulation. Personality and Individual Differences, 47(5), 481-486.

Read, S., Aunola, K., Feldt, T., Leinonen, R., y Ruoppila, I. (2005). The relationship between generalized resistance resources, sense of coherence, and health among Finnish people aged 65-69. European Psychologist, 10(3), 244-253.

Robles, R., y Páez, F. (2003). Estudio sobre la traducción al español y las propiedades psicométricas de las escalas de afecto positivo y negativo (PANAS). Salud Mental, 26(1), 69-75.

Rodríguez-Carvajal, R., Moreno-Jiménez, B., y Garrosa, E. (2006). Cuestionario de Regulación Emocional. Versión española. Autorizado por los autores de la versión original en Inglés (Gross y John, 2003). Madrid: Universidad Autónoma de Madrid.

Russell, J. A., y Carroll, J.M. (1999). On the bipolarity of positive and negative affect. Psychological Bulletin, 125(1), 3-30.

Salanova, M., Agut, S., y Peiró, J. M. (2005). Linking Organizational Resources and Work Engagement to Employee Performance and Customer Loyalty: The Mediation of Service Climate. Journal of Applied Psychology, 90(6), 1217-1227.

Salanova, M., y Schaufeli, W. (2009). El engagement en el trabajo. Cuando el trabajo se convierte en pasión. Madrid: Alianza.

Salanova, M., Llorens, S., y Schaufeli, W. B. (2011). "Yes, I Can, I Feel Good, and I Just Do It!" On Gain Cycles and Spirals of Efficacy Beliefs, Affect, and Engagement. Applied Psychology, 60(2). 255-285.

Salanova,M., Schaufeli, W. B., Llorens,S., Pieró, J. M., y Grau, R.(2001). Desde el"burnout"al"engamement": Una nueva perspectiva. Revista de Psicología del Trabajo y de las Organizaciones,16(2), 117-134.

Schaufeli, W. (2004). The Future of Occupational Health Psychology. Applied Psychology: An International Review, 53(4), 502-517.

Schaufeli, W. B., y Bakker, A. B. (2001). Werk en welbevinden: naar een positieve benadering in de Arbeids en Gezondheidspsychologie [Work and well-being: towards a positive approach in Occupational Health Psychology]. Gedrag \& Organisatie, 14, 229-253.

Schaufeli, W. B., y Bakker, A. B. (2004). Job demands, job resources, and their relationship with burnout and engagement: a multi-sample study. Journal of Organizational Behavior, 25(3), 293-315.
Schaufeli, W. B., Salanova, M., González-Romá, V., y Bakker, A. (2002). The measurement of engagement and burnout: a two simple confirmatory factor analytic approach. Journal of Happiness Studies, 3(1), 71-92.

Seligman, M. E. P., y Csikszentmihalyi, M. (2000). Positive psychology: An introduction. American Psychologist, 55(1), 5-14.

Shirom, A. (2011). Vigor as a positive affect at work: conceptualizing vigor, its relations with related constructs, and its antecedents and consequences. Review of General Psychology, 15(1), 50-64.

Strümpfer, D. J. W. (2003). Resilience and burnout: A stitch that could save nine. South African Journal of Psychology, 33(2), 69-79.

Strümpfer, D. J. W., y de Bruin, G. P. (2009). Antonovsky's Sense of Coherence and job satisfaction: Meta-analysis of South African data. South African Journal of Industrial Psychology, 35(1), 172-174.

Strümpfer, D. J. W., Gouws, J. F., y Viviers, M. R. (1998). Antonovsky's Sense of Coherence Scale related to negative and positive affectivity. European Journal of Personality, 12(6), 457-480.

Trufelli, D., Bensi, C., García, J., Narahara, J., Abrao, M., Diniz, R., Da Costa Miranda, V., Soares, H. y Del Giglio, A. (2008). Burnout in cancer professionals: a systematic review and meta-analysis. European Journal of Cancer Care, 17, 524-531.

Turner, N., Barling, J., y Zacharatos, A. (2002). Positive psychology at work. En C.R Snyder y S. López. (Eds.), The handbook of positive psychology (pp. 715-730). Oxford: Oxford University Press.

Van der Colff, J. J., y Rothmann, S. (2009). Occupational stress, sense of coherence, coping, burnout and work engagement of registered nurses in South Africa. South African Journal of Industrial Psychology, 35(1), 1-10.

Vecina, M. L., y Chacón, F. (2005). Positive Emotions in Volunteerism. The Spanish Journal of Psychology, 8(1), 30-35.

Vecina, M. L., Chacón, F. y Sueiro, M. J. (2009). Satisfacción en el voluntariado: estructura interna y relación con la permanencia en las organizaciones. Psicothema, 21 (1), 112-117.

Vecina, M. L., Chacón, F., Sueiro, M. y Barrón, A. (2012). Volunteer Engagement: Does Engagement Predict the Degree of Satisfaction among New Volunteers and the Commitment of Those who have been Active Longer? Applied Psychology, 61(1), 130-148.

Volanen, S., Lahelma, E., Silventoinen, K., y Suominen, S. (2004). Factors contributing to sense of coherence among men and women. European Journal of Public Health, 14(3), 322-330.

Wallace, J. C., Edwards, B. D., Shull, A., y Finch, D. M. (2009). Examining the consequencesin the tendency to suppress and reappraise emotions on task-related job performance. Human Performance, 22(1), 23-43.

Watson, D., Clark, L. A., y Tellegen, A. (1988). Development and validation of brief measures of positive and negative affect: The PANAS scales. Journal of Personality and Social Psychology, 54(6), 1063-1070.

Whippen, D. A., y Canellos, G. P. (1991). Burnout syndrome in the practice of oncology: results of a random survey of 1,000 oncologists. Journal of Clinical Oncology, 9(10), 1916-1920.

Wiesmann, U., y Hannich, H. (2010). A salutogenic analysis of healthy aging in active elderly persons. Research on Aging, 32(3), 349-371.

Xanthopoulou, D., Bakker, A., Demerouti, E., y Schaufeli, W. B. (2007). The role of personal resources in the Job Demands-Resources model. International Journal of Stress Management, 14(2), 121-141.

(Artículo recibido: 8-3-2012; revisado: 26-11-2012, aceptado: 27-11-2012) 